\title{
ON 2-TRANSITIVE GROUPS IN WHICH THE STABILIZER OF TWO POINTS FIXES ADDITIONAL POINTS $\dagger$
}

\author{
WILLIAM M. KANTOR
}

\section{Introduction}

Let $\Gamma$ be a 2-transitive group of finite degree $v$ such that the stabilizer $\Gamma_{x y}$ of the distinct points $x$ and $y$ fixes precisely $k$ points, where $2<k<v$. The only known non-solvable groups with this property are of the following types: (i) a 2-transitive collineation group of $\operatorname{PG}(d, 2)$ for some $d$; (ii) a 2-transitive collineation group of $\operatorname{AG}(d, k)$ for some $d$; and (iii) $\operatorname{P\Gamma L}(2,8)$ in its representation of degree 28 . We shall prove some characterizations of groups of these types. Let $X$ be the set of fixed points of $\Gamma_{x y}$ and $\Gamma_{x}=N\left(\Gamma_{x y}\right)$ the global stabilizer of $X$. Our main result (Theorem 5.1) states that $\Gamma$ is of the form (i) or (ii) provided that $\Gamma_{x} \cap \Gamma_{X}$ is transitive on the points not in $X$. This generalizes a result of Ito [13] (see [4; pp. 47-48]) which assumes that $\Gamma_{x y}$ is transitive and regular on the points not in $X$. Our approach is, however, quite different from Ito's. When $k$ is even we use a result of Bender [2]. When $k$ is odd a result of Hall [9] is used in an unexpected way. The case where $\Gamma_{x y}$ is not regular on the points it moves is deduced from the case where this group is regular by means of a result of Glauberman [7].

This research was begun in order to obtain a proof of the above mentioned result of Ito [13]. I am indebted to Prof. Ito for several useful discussions concerning results of the type presented here. I am also grateful to Dr. F. Buekenhout for pointing out errors in an earlier version of this paper.

\section{Preliminaries}

If $\Delta$ is a subset of a group $\Gamma, N_{\Gamma}(\Delta)$ and $C_{\Gamma}(\Delta)$ (or simply $N(\Delta)$ and $C(\Delta)$ ) are its normalizer and centralizer in $\Gamma$, respectively. $O(\Gamma)$ is the largest normal subgroup of $\Gamma$ of odd order.

We shall consider the following situations.

$\left(^{*}\right) \Gamma$ is a 2-transitive group of finite degree $v, x$ and $x^{\prime}$ are distinct points, and $\Gamma_{x x^{\prime}}$ fixes precisely $k$ points, where $2<k<v$.

$(* *) \Gamma$ satisfies $\left(^{*}\right)$ and $\Gamma_{x x^{\prime}}$ acts regularly (but not necessarily transitively) on the points it moves.

Let $\Gamma$ satisfy $\left({ }^{*}\right)$. We shall use the following notation. $X$ is the set of points fixed by $\Pi=\Gamma_{x x^{\prime}} . N(\Pi)=\Gamma_{X}$ is the global stabilizer of $X . \mathscr{D}(\Gamma)$ consists of the points permuted by $\Gamma$ and the sets $X^{\gamma}, \gamma \in \Gamma$. Two distinct points are in exactly one such set: $\mathscr{D}(\Gamma)$ is a design with $\lambda=1$ ([5], Chapter 2). The sets $X^{y}$ will be called lines. $\Pi=\Gamma(X)$ is the pointwise stabilizer of the line $X$. There are $r=(v-1) /(k-1)$ lines on $x . \Gamma(x)$ is the linewise stabilizer of this set, and $\Gamma_{x}=\Gamma_{x} / \Gamma(x)$ is the permutation group induced by $\Gamma_{x}$ on these lines.

By $\left(^{*}\right), \Gamma_{X}$ acts on $X$ as a sharply 2-transitive group. In particular, $k$ is a prime power.

Received 10 April, 1969; revised 5 December, 1969.

† Research supported in part by NSF Grant GP 6539.

[J. LoNDON MATH. Soc. (2), 5 (1972), 114-122] 
3. $k$ even

Let $\Gamma$ satisfy $\left({ }^{* *}\right)$ with $k$ even. Then $k=2^{e}, e \geqslant 2$.

Lemma 3.1. $v$ is even, $k \mid(r-1)$, and each involution in $\Gamma$ fixes $v / k$ pairwise disjoint lines.

Proof. Suppose that $v$ is odd, and let $\sigma$ be an involution in $\Gamma_{x}$. As $v-k$ is odd, by (**) $\sigma$ fixes no point other than $x$. If $y \neq x$ then $\sigma$ fixes the line through $y$ and $y^{\sigma}$. As $k$ is even, no two such lines meet. Thus, $\sigma$ fixes $(v-1) / k$ pairwise disjoint lines. On the other hand, by a result of Bender [2], either $\Gamma$ has a normal subgroup similar to $\operatorname{PSL}(2, q), \operatorname{Sz}(q)$ or $\operatorname{PSU}(3, q)$ in its usual representation, where $q$ is even, or $v$ is a power of a prime $p$ and $\Gamma$ has a regular normal elementary abelian $p$-subgroup. In the former case $(* *)$ cannot hold. In the latter case the number of fixed points of an element of $\Gamma(X)$ is a power of $p$. As $k \mid(v-1)$ we must have $\Gamma(X)=1$, a contradiction. Thus, $v$ is even, and it is easy to complete the proof.

Let $\Sigma$ be a Sylow 2-subgroup of $\Pi$. If $\Sigma=1$ then $\Gamma$ is solvable [1]. Assume that $\Sigma \neq 1$, and let $m$ be the number of involutions in $\Pi$.

LEMMA 3.2. (i) $m \leqslant k-1$, with equality if and only if $\Gamma_{y X}$ is transitive on $X$ whenever $y \notin X$. (ii) If $m>1$ then $\Sigma$ contains distinct commuting involutions.

Proof. If $x \neq x^{\prime} \in X$ and $y \notin X$, let $n\left(x, x^{\prime} ; y\right)$ be the number of involutions of the form $(y)\left(x x^{\prime}\right) \ldots$. Count in two ways the ordered quadruples $\left(x, x^{\prime}, y, \gamma\right)$ with $\gamma$ an involution of the form $(y)\left(x x^{\prime}\right) \ldots$ and $x, x^{\prime}, y$ non-collinear:

$$
v(v-1) \sum_{y \notin X} n\left(x, x^{\prime} ; y\right)=\frac{v(v-1)}{k(k-1)} m .(v-k) k,
$$

or

$$
\sum_{y \notin X} n\left(x, x^{\prime} ; y\right)=m(r-1)
$$

whenever $x \neq x^{\prime} \in X$.

(i) This follows from the inequality $n\left(x, x^{\prime} ; y\right) \leqslant 1$.

(ii) Let $x_{i}(i=1, \ldots, k-1)$ be the points of $X-\{x\}$. As

$$
\sum_{y \notin X} \sum_{i} n\left(x, x_{i} ; y\right)=m(r-1) .(k-1)>v-k,
$$

there is a point $y \notin X$ and there are distinct integers $i$ and $j$ such that $\Gamma_{y X}$ has involutions (y) $\left(x x_{1}\right) \ldots$ and $(y)\left(x x_{j}\right) \ldots$ These generate a group of order 4 fixing some line $X^{*}$ pointwise as $v-k$ is even.

LEMMA 3.3. Let $\Gamma$ be a non-solvable group satisfying $\left(^{* *}\right)$ such that the Sylow 2-subgroups of $\Gamma_{x x^{\prime}}$ have only one involution. If $k$ is even then $v=28$ and $\Gamma \approx \operatorname{P} \Gamma L(2,8)$.

Proof. By Lemma 3.2 (ii), $\Pi$ has a unique involution $\sigma$. As $\sigma$ fixes a line $X^{*} \neq X$ it centralizes the involution $\tau$ in $\Gamma\left(X^{*}\right)$. As $C_{\Pi}(\tau)$ fixes $X^{*}$ and acts regularly on $X^{*}$, $C_{\Pi}(\tau)=\langle\sigma\rangle$. We may assume that $\tau$ normalizes $\Sigma$.

By the Frattini argument, $\Gamma_{\boldsymbol{X}}=\Pi N(\Sigma)$, so that $N(\Sigma)$ is 2-transitive on $X$. So $N(\Sigma) / C(\Sigma)(N(\Sigma) \cap \Pi)$ is a homomorphic image of a sharply 2-transitive group of degree $k$ and of $N(\Sigma) / C(\Sigma) \Sigma$. However, $N(\Sigma) / C(\Sigma) \Sigma$ is isomorphic to a group of outer automorphisms of $\Sigma$, hence is a 2 -group or has order 3 or 6 . It follows that 
$|N(\Sigma) / C(\Sigma) \Sigma|=1$ or 3. Then $\tau \in C(\Sigma) \Sigma$, so that $\tau=\gamma \sigma_{1}$ with $\gamma \in C(\Sigma)$ and $\sigma_{1} \in \Sigma$. Now $\sigma_{1} \in C_{\Pi}(\tau)=\langle\sigma\rangle, \tau \in C(\Sigma)$, and hence $\Sigma \leqslant C(\tau)=\langle\sigma\rangle$.

Thus, $|\Sigma|=2$, so that $\Pi=\Sigma O(\Pi)$. As $C_{o(\Pi)}(\tau)=1, \tau$ inverts $O(\Pi)$. Let $\tau^{\prime}$ be a conjugate of $\tau$ by an element of $\Gamma_{x X}-\Pi$. Then $\tau \tau^{\prime}$ is fixed point free on $X$ and centralizes $O(\Pi)$. Also, $\tau \tau^{\prime} \in C(\Pi)$. As $N(\Pi)=\Gamma_{X}$ is 2-transitive on $X$, it follows that $C(\Pi)$ is transitive on $X$. Consequently, $C(\Pi)$ contains a Sylow 2-subgroup of $\Gamma_{x}$, and hence also an involution $\tau^{\prime \prime}$ conjugate to $\tau$ in $\Gamma_{X}$. Then $\tau^{\prime \prime}$ is an element of $C(\Pi)$ inverting $O(\Pi)$, so that $O(\Pi)=1$ and $\Pi=\Sigma$. The lemma now follows from a result of Ito [12].

THEOREM 3.4. If $\Gamma$ is a non-solvable group satisfying $\left({ }^{* *}\right)$ with $k$ even, then either $v=28$ and $\Gamma \approx \mathrm{P} \Gamma \mathrm{L}(2,8)$, or $\mathscr{D}(\Gamma)$ is a desarguesian affine plane and $\Gamma$ contains all elations.

Proof. By Lemma 3.3 we may assume that the Sylow 2-subgroups of $\hat{\Gamma}_{x}$ have more than one involution. If $x \in Y \neq X$ then $\left(\hat{\Gamma}_{x}\right)_{X Y}$ has odd order. It thus follows from a result of Bender [2] that $\Gamma_{x}$ has a normal subgroup $\Delta$ containing $\Gamma(x)$ such that $\widehat{\Delta}=\Delta / \Gamma(x)$ is isomorphic to $\operatorname{PSL}\left(2,2^{f}\right), \operatorname{Sz}\left(2^{f}\right)$ or $\operatorname{PSU}\left(3,2^{f}\right)$ for some integer $f$. Moreover, $\Delta$ acts on the lines on $x$ in its usual 2-transitive representation, and $s=\left|\Gamma_{x} / \Delta\right|$ is odd.

The Sylow 2-subgroup $\Sigma$ of $\Gamma_{x X}$ is sharply transitive on the lines $\neq X$ on $x$. Thus, $\Gamma_{x X}=\Sigma \Gamma_{X Y}$. As $\Gamma_{x X}$ is transitive on $X-\{x\}$, so is $\Gamma_{X Y}$. Then $\Gamma_{X Y}$ is also transitive on $Y-\{x\}$. It follows that $\Gamma_{x X}$ is transitive on the points not on $X$.

Let $y \notin X$. Then $\left|\Gamma_{y X}: \Gamma_{x y X}\right|=\left|\Gamma_{X}: \Gamma_{x X}\right|\left|\Gamma_{x X}: \Gamma_{x y X}\right| /\left|\Gamma_{X}: \Gamma_{y X}\right|=k$, so that $\Gamma_{y X}$ is transitive on $X$. The Sylow 2-subgroup $K$ of $\Gamma_{y X}$ is elementary abelian of order $k$. As $v-k-1$ is odd, $K$ fixes a line $X^{*} \neq X$ pointwise. By Lemma 3.2 (i), $K$ contains all of the $k-1$ involutions in $\Gamma\left(X^{*}\right)$. However, a Sylow 2-subgroup of $\Delta$ has precisely $2^{f}-1$ involutions. Thus, $e=f$. Then $r=2^{\varepsilon e}+1$, where $\varepsilon=1,2$ or 3 if $\hat{\Delta} \approx \operatorname{PSL}\left(2,2^{e}\right)$, $\mathrm{Sz}\left(2^{e}\right)$ or $\operatorname{PSU}\left(3,2^{e}\right)$, respectively.

If $r=2^{e}+1$ then $\mathscr{D}(\Gamma)$ is an affine plane of order $k$ in which each line is the axis of $k$ elations. Consequently, $\mathscr{D}(\Gamma)$ is desarguesian $[5 ;$ p. 126]. We now assume that $\varepsilon=2$ or 3 .

If $\varepsilon=3$ then, for $d=1$ or $1 / 3$,

$$
d\left(2^{2 e}-1\right) s|\Gamma(x)|=\left|\Gamma_{X Y}\right|=\left(2^{e}-1\right)\left|\Gamma(X)_{Y}\right| \mid\left(2^{e}-1\right)^{2},
$$

a contradiction. Thus, $\varepsilon=2$. In particular, $k=2^{e} \geq 8$.

There is an involution $\sigma \in \Pi$ centralized by $K$. Let $K^{*}$ be the Sylow 2-subgroup of $\Gamma_{x X^{*}}$. Then $\sigma \in K^{*}$ implies that $K^{*} \leqslant \Pi$.

Suppose that $1 \neq \alpha \in \Gamma(x)$. Then $[\alpha, \Pi] \subseteq[\Gamma(x), \Pi]=1[14]$. Let $\alpha^{\prime} \neq \alpha$ be conjugate to $\alpha$ by an element of $K$. Then $\alpha^{-1} \alpha^{\prime}$ centralizes $\Pi$ and is fixed point free on $X$. As $|\Sigma|=r-1>k, \alpha^{-1} \alpha^{\prime}$ is fixed point free. There is an element $1 \neq \tau \in K$ such that $\alpha^{-1} \alpha^{\prime} \tau \in \Pi$. Then $\alpha^{-1} \alpha^{\prime}$ centralizes $\alpha^{-1} \alpha^{\prime} \tau$ and hence also $\tau$, so that $\alpha^{-1} \alpha^{\prime}$ fixes $X^{*}$. There is thus an involution $\sigma_{1} \in K^{*}$ such that $\alpha^{-1} \alpha^{\prime} \sigma_{1}$ fixes a point of $X^{*}$. As $\alpha^{-1} \alpha^{\prime} \sigma_{1}$ centralizes $\Sigma$ it fixes at least $|\Sigma|>k$ points but is fixed point free on $X$, a contradiction. Thus, $\Gamma(x)=1$. It follows that $\Delta_{X Y}$ is cyclic. 
We now show that $\Gamma(X)_{Y}=1$, and hence that $\Pi=\Sigma$ has order $r-1$. For, $\Gamma(X)_{Y^{\prime}} \Gamma(Y)_{X}$ and $\Delta_{X Y}$ are normal in $\Gamma_{X Y}$. There is an element of $\Delta$ interchanging $\Gamma(X)_{Y}$ and $\Gamma(Y)_{X}$ while normalizing the cyclic group $\Delta_{X Y}$. Thus $\Gamma(X)_{Y} \cap \Delta_{X Y}=1$, so $\Gamma(X)_{Y}$ centralizes $\Delta_{X Y}$. From the structure of $\Gamma_{x}$ it follows that $\Gamma(X)_{Y}=1$.

Let $1 \neq \tau \in K$ invert $c$ elements of $\Pi$. If $\Gamma$ has $c^{\prime}$ fixed point free involutions, then

$$
\begin{gathered}
\frac{v(v-1)}{k} \leq c^{\prime}+\frac{v(v-1)}{k(k-1)} \cdot(k-1)=r(k-1)+v(v-1) c, \\
c>v / k-1=(k-1)(r-1) / k>\frac{3}{4}(r-1)=\frac{3}{4}|\Pi| .
\end{gathered}
$$

A result of G. A. Miller [10; p. 60, Ex. 12] now implies that $\tau$ inverts $\Pi$. As $\Pi$ is non-abelian, this is a contradiction.

Corollary 3.5. Under the hypotheses of Theorem 3.4, if also $\Gamma_{x}$ is 2-transitive on the set of distinct sets $X^{\gamma}, \gamma \in \Gamma_{x}$, then $\mathscr{D}(\Gamma)$ is a desarguesian affine plane and $\Gamma$ contains all elations.

Proof. Theorem 3.4 and Huppert [11].

\section{4. $k$ odd}

Let $\Gamma$ satisfy $(* *)$ with $k$ odd. $r=(v-1) /(k-1)$ implies that $v$ is odd. $k=p^{e}$ where $p$ is prime. Let $\Pi$ have $m$ involutions.

LeMMA 4.1. Let $\sigma$ be an involution in $\Gamma$.

(i) If $\sigma$ fixes only one point $x$ then $\sigma \in \Gamma(x)$.

(ii) If $\sigma$ fixes at least two points it fixes a unique line $X$ pointwise. Moreover, $\sigma$ fixes precisely $r-1$ lines $\neq X$, and each of these meets $X$.

Proof. (i) If $y \neq x$ then $\sigma$ fixes the line through $y$ and $y^{\sigma}$. As $k$ is odd, $\sigma$ fixes a point of this line, which must be $x$. It follows that $\sigma$ fixes all lines on $x$.

(ii) The existence and uniqueness of $X$ are immediate. If $y \neq x$ then $\sigma$ fixes the line through $y$ and $y^{\sigma}$, and as $k$ is odd this line meets $X$. It follows that $\sigma$ fixes $(v-k) /(k-1)=r-1$ lines $\neq X$, each meeting $X$.

Lemma 4.2. $m \leq k$, and either $m=0, m=1$ or $m \geqslant p$. Moreover, the following are equivalent if $m>0$.

(i) $m=k$;

(ii) $\left|\Gamma(X)_{Y}\right|$ is even whenever $|X \cap Y|=1$;

(iii) $\Gamma_{y X}$ is transitive on $X$ for all $y \notin X$; and

(iv) $\Gamma_{y X}$ is transitive on $X$ for some $y \notin X$.

The proof is similar to that of Lemma 3.1.

Lemma 4.3. Let $\Gamma(x)$ have even order.

(i) If $\Pi$ has even order then $\mathscr{D}(\Gamma)$ is $\mathrm{PG}(2,2)$ or an affine translation plane. 
(ii) Either $\Gamma$ has a non-trivial regular normal elementary abelian p-subgroup or $\mathscr{D}(\Gamma)$ is $\mathrm{PG}(2,2)$.

Proof. Let $\Pi$ have even order. Let $\sigma$ be an involution in $\Pi$ fixing a line $Y \neq X$ on $x$, and $\delta \in \Gamma(x)$ an involution in neither $\Gamma(X)$ nor $\Gamma(Y)$. Then $\sigma=\delta$ on $Y$. If $\sigma$ fixes a third line $Z$ on $x$, then $\delta$ must fix $Z$ pointwise, and taking $\sigma \in \Gamma(x) \cap \Pi$, we find that $r=3$. If $r>3$, it follows that $\sigma$ fixes at most $k$ lines $\neq X$. Then $r-1 \leqslant k$ by Lemma 4.1 (ii), and it is easy to see that $\mathscr{D}(\Gamma)$ is a translation plane.

Suppose now that $\Pi$ has odd order. By a result of Bender [2], either $\Gamma$ has a normal subgroup similar to $\operatorname{PSL}(2, q), \operatorname{Sz}(q)$ or $\operatorname{PSU}(3, q)$ in its usual representation, where $q$ is even, or $\Gamma$ has a regular normal elementary abelian $p^{\prime}$-subgroup of order $\mathrm{p}^{\prime d}$, where $p^{\prime}$ is prime. The first possibility cannot occur. In the second case, the number of fixed points of each element of $\Gamma(X)$ is a power of $p^{\prime}$. Thus, $p^{\prime}=p$.

LEMMA 4.4. Let $\Gamma(x)$ have odd order.

(i) If $\sigma$ is an involution in $\Pi$ and $x \in X$, then $\sigma$ fixes precisely $1+(r-1) / k$ lines on $x$. Moreover, $C(\sigma)$ is transitive on $X$.

(ii) If $|X \cap Y|=1$ then $\Gamma_{X Y}$ has precisely 0,1 or 3 involutions.

Proof. (i) Set $S(\sigma)=\{x \in X \mid \sigma$ fixes at least 2 lines on $x\}$. As $|\Gamma(x)|$ is odd, $|S(\sigma)|>1$ by Lemma 4.1 (ii). Assume that $|S(\sigma)|<k$. By Lemma 4.2 there are at most $k$ distinct sets $S(\sigma)^{\gamma}, \gamma \in \Gamma_{X}$. Then $1<\left|\Gamma_{X}: \Gamma_{X S(\sigma)}\right| \leq k$ implies that $\Gamma_{X S(\sigma)}=\Gamma_{x X}$ for some $x \in X$. There are thus $k$ sets $S(\sigma)^{\gamma}, \gamma \in \Gamma_{X}$, hence also $k$ involutions in $\Gamma(X)$; moreover, $S(\sigma)=X-\{x\}$. However, if $x \neq x^{\prime} \in X$ then $\sigma$ fixes some line $Y \neq X$ on $x^{\prime}$. As $\sigma$ normalizes $\Gamma(Y)$ it centralizes an involution $\tau \in \Gamma(Y)_{X}$. Then $S(\sigma)^{\tau}=S(\sigma)$, while $\tau$ moves $x$, a contradiction.

Thus, $S(\sigma)=X$. If $x \in X$ and $\sigma$ fixes the line $Y \neq X$ on $x$, then $\sigma$ normalizes $\Gamma(Y)$, and hence centralizes an involution $\tau$ in $\Gamma(Y)$. Clearly $\tau \in C(\sigma) \cap \Gamma(Y)_{X}$. It follows that $C(\sigma)$ is transitive on $X$. Consequently, each point of $X$ is on the same number of fixed lines of $\sigma$. As $\sigma$ fixes $r-1$ lines $\neq X$, each point of $X$ is on $(r-1) / k$ of these lines.

(ii) Let $\sigma$ and $\tau$ be distinct involutions in $\Gamma_{X Y}$. First assume that $\sigma \in \Gamma(X)_{Y}$ and $\tau \in \Gamma(Y)_{X}$. Then $[\sigma, \tau] \in \Gamma(X)_{Y} \cap \Gamma(Y)_{X}=1$, so $\sigma \tau$ is an involution. If $\alpha$ is an involution in $\Gamma_{X Y}-\Gamma(X)_{Y}$ then $\alpha=\tau$ on $X$, so $\alpha \tau \in \Gamma(X)_{Y}$. As $\tau$ centralizes $\Gamma(X)_{Y}$, $\alpha \tau$ is 1 or an involution, that is, $\alpha \tau=1$ or $\sigma$.

We may now assume that $\sigma$ is in neither $\Gamma(X)_{Y}$ nor $\Gamma(Y)_{X}$. As $\sigma$ and $\tau \sigma \tau$ agree on $X$ and $Y, \sigma$ and $\tau$ commute. Similarly, $\tau$ must be in $\Gamma(X)_{Y}$ or $\Gamma(Y)_{X}$, say $\Gamma(Y)_{X}$. Then $\sigma \tau \in \Gamma(X)_{Y}$ and $\sigma \tau$ is an involution. We have now reduced to the first situation.

Theorem 4.5. Let $\Gamma$ satisfy $\left({ }^{*}\right)$ with $k$ odd. Assume that $\Gamma_{x}$ is 2-transitive on the set of distinct sets $X^{\gamma}, \gamma \in \Gamma_{x}$. Then either $\mathscr{D}(\Gamma)$ is a desarguesian affine plane and $\Gamma$ contains all elations, $\mathscr{D}(\Gamma)$ is $\operatorname{PG}(2,2)$ and $\Gamma$ is $\operatorname{PSL}(3,2)$, or $\mathscr{D}(\Gamma)$ is $\operatorname{PG}(3,2)$ and $\Gamma \approx A_{7}$.

Proof. By hypothesis, $\hat{\Gamma}_{x}$ is 2-transitive.

Case 1. $|\Gamma(x)|$ is even. If $|\Pi|$ is even then, by Lemma 4.4 (i), we may assume that $\mathscr{D}(\Gamma)$ is an affine translation plane. As $r-1=k$, a Sylow $p$-subgroup of $\Gamma_{x}$ fixes some line $X$ on $x$ pointwise and is transitive on the remaining lines on $x$. It follows that $\mathscr{D}(\Gamma)$ is desarguesian [5; p. 126]. 
Suppose now that $|\Pi|$ is odd. By Lemma 4.4 (ii), $\Gamma$ has a non-trivial regular normal elementary abelian $p$-subgroup. As $k|v=1+r(k-1), k|(r-1)$.

By hypothesis, $(r-1)|| \Gamma_{x} \mid$. Let $\Lambda$ be a Sylow $p$-subgroup of $\Gamma_{x X}$. Then $\Lambda \leq \Gamma(X)$ and $k|| \Lambda \mid$. We may regard $\Gamma$ as a collineation group of the affine space $\operatorname{AG}(d, p)$, where $v=p^{d}$. $X$ is an $e$-subspace, where $k=p^{e}$. $\Lambda$ fixes an $(e+1)$-subspace containing $X$ and acts faithfully on this subspace. It follows that $|\Lambda|=k$ and $\Lambda$ is elementary abelian.

We also note that, by [14], if $x \in X$ then $\Gamma(x) \cap \Gamma(X)=1$. Suppose that $O\left(\hat{\Gamma}_{x}\right) \neq 1$. As $O\left(\hat{\Gamma}_{x}\right)$ is solvable by the Feit-Thompson Theorem [6], $\hat{\Gamma}_{x}$ has a regular normal elementary abelian subgroup $\hat{\Phi}$ of order $r$, where $\hat{\Phi}=\Phi / \Gamma(x), \Gamma_{x} \geqslant \Phi \geqslant \Gamma(x)$. If $y \neq x$ and $Y$ is the line joining $x$ and $y$, then $\Phi_{y} \leqslant \Gamma(x) \cap \Gamma(Y)=1$. $\Phi$ is thus a Frobenius complement, so that $\hat{\Phi}$ is cyclic and $r$ is prime. Then $\left(\hat{\Gamma}_{x}\right)_{X}$ is cyclic. However, $\Lambda \cap \Gamma(x) \leqslant \Gamma(X) \cap \Gamma(x)=1$, so that $\Lambda$ is isomorphic to a subgroup of $\left(\hat{\Gamma}_{x}\right)_{X}$. Then $\Lambda$ is cyclic of order $k$. As $\Lambda$ is known to be elementary abelian, $k=p$. It follows that $\hat{\Gamma}_{x}$ is a sharply 2 -transitive collineation group of $\operatorname{PG}(d-1, p)$. This can happen only when $d=2$ and $p=3$, and then $\mathscr{D}(\Gamma)$ is $\operatorname{AG}(2,3)$.

We now assume that $O(\hat{\Gamma})_{x}=1$. As $\Pi$ has odd order, $\Gamma_{x}$ has exactly one involution, and this belongs to $\Gamma(x)$. Consequently, $\Gamma_{x}$ has cyclic or generalized quaternion Sylow 2-subgroups. Then $\hat{\Gamma}_{x}$ has cyclic or dihedral Sylow 2-subgroups. By the transfer theorem of Burnside and a result of Gorenstein and Walter [8], either $\hat{\Gamma}_{x} \approx A_{7}$ or $\hat{\Gamma}_{x}$ is isomorphic to a subgroup of $\operatorname{P\Gamma L}(2, q)$ containing $\operatorname{PSL}(2, q)$, where $q$ is an odd prime power.

If $\hat{\Gamma}_{x} \approx A_{7}$ then $r=7$ or 15 , since $\hat{\Gamma}_{x}$ is 2-transitive. If $r=7$, then, as $k \mid(r-1)$ we must have $k=3$, so that $v=15$ is not a power of 3 . Similarly, if $r=15$ then $k=7$ and $r=91$.

Thus, $\hat{\Gamma}_{\boldsymbol{x}}$ is isomorphic to a subgroup of $\operatorname{P\Gamma L}(2, q)$ containing $\operatorname{PSL}(2, q)$. By a result of Lüneburg [5; p. 196], if $r \neq q+1$ then $r=5,6,7$ or 11. Once again, $k \mid(r-1)$ and $v$ a power of $p$ imply that $k=5$ and $v=5^{2}$, so that $\mathscr{D}(\Gamma)$ is $\operatorname{AG}(2,5)$. We may thus assume that $r=q+1$. Then $k \mid(r-1)$ implies that $q$ is a power of $p$. As $q|| \Lambda \mid=k$, it follows that $\mathscr{D}(\Gamma)$ is a desarguesian affine plane [5, p. 126].

Case 2. $|\Gamma(x)|$ is odd. By Lemma 4.3, each involution in $\Gamma(X)$ fixes $1+(r-1) / k$ lines on each point of $X$, and any two distinct intersecting lines are fixed by precisely 3 involutions. Count the ordered triples $(X, Y, \gamma)$ consisting of distinct lines $X$ and $Y$ on $x$ and an involution $\gamma \in \Gamma_{X Y}$ :

Thus, $r=2 k+1$.

$$
r k .(1+(r-1) / k)((r-1) / k)=r(r-1) \cdot 3 \text {. }
$$

Clearly $r>3$. Define a Steiner triple system $\mathscr{S}(x)$ as follows. The "points" of $\mathscr{S}(x)$ are the lines on $x$. If $X$ and $Y$ are distinct lines on $x$, and $\sigma$ and $\tau$ are the involutions in $\Gamma(X)_{Y}$ and $\Gamma(Y)_{X}$, respectively, then the line fixed pointwise by the involution $\sigma \tau$ is the third member of the triple determined by $X$ and $Y$.

$\hat{\Gamma}_{x}$ is an automorphism group of $\mathscr{S}(x)$. Moreover, $\mathscr{S}(x)$ has the property that each triple is fixed pointwise by precisely 3 involutions, commuting with one another and fixing only the 3 points of this triple. By a result of Hall [9, Theorem 3.2], any 3 points of $\mathscr{S}(x)$, not forming a triple, generate a subsystem $\operatorname{PG}(2,2)$ or $\operatorname{AG}(2,3)$. As the 3 involutions commute, we must always have PG(2,2). By the Veblen and Young axioms [16], $\mathscr{S}(x)$ consists of the points and lines of a projective space 
$\operatorname{PG}(d, 2)$. As the involutions fix only the 3 points of a projective line, $(d+1) / 2 \leqslant(1+1) / 2$. If $d=2$ then $v=15, \Gamma \approx A_{7}$, and $\mathscr{D}(\Gamma)$ is $\mathrm{PG}(3,2)$. If $d=3$ then $r=15$ and $\hat{\Gamma}_{x} \approx A_{7}$. Then $\left(\hat{\Gamma}_{x}\right)_{x} \approx \operatorname{PSL}(3,2)$ and $\Gamma_{x x} / \Gamma(x) \unrhd \Gamma(x) \Pi / \Gamma(x)$ imply that $\Pi \leqslant \Gamma(x)$ or $\Gamma_{x X}=\Gamma(x) \Pi$. The first possibility is clearly impossible. The second yields that $\Gamma_{x x} / \Pi \approx \Gamma(x) / \Gamma(x) \cap \Pi$. As $\Gamma(x)$ has odd order, this is also a contradiction.

COROLlary 4.6. Let $\Gamma$ satisfy $\left({ }^{* *}\right)$. If $k$ is odd and $\Gamma_{x x^{\prime}}$ has at least $k$ involutions, then either $\mathscr{D}(\Gamma)$ is a desarguesian affine plane and $\Gamma$ contains all elations, $\mathscr{D}(\Gamma)$ is $\mathrm{PG}(2,2)$, or $\mathscr{D}(\Gamma)$ is $\mathrm{PG}(3,2)$ and $\Gamma \approx A_{7}$.

Proof. By Lemma 4.2, $\Pi$ has $k$ involutions and $\Pi_{Y}$ has even order whenever $|X \cap Y|=1$. If $\Gamma(x)$ has even order then, by Lemma 4.4 (i), we may assume that $\mathscr{D}(\Gamma)$ is an affine translation plane. As $\Pi$ has $k=r-1$ involutions, Gleason's lemma $[5 ;$ p. 191] implies that $\Pi$ is transitive on the lines $\neq X$ on $x$. As in Theorem $4.5, \mathscr{D}(\Gamma)$ is a desarguesian affine plane.

If $\Gamma(x)$ has odd order, the result follows from precisely the same argument as that used in Theorem 4.5, Case 2.

COROLlary 4.7. Let $\Gamma$ satisfy (**) with $k$ prime. If $\Gamma_{x x^{\prime}}$ has more than one involution then $\Gamma$ is similar to a collineation group of a desarguesian affine plane containing all elations, $\operatorname{PSL}(3,2)$ in its usual representation, or $A_{7}$ in its 2-transitive representation of degree 15.

Proof. Lemma 4.2 and Corollary 4.6.

\section{The non-regular case}

THeOReM 5.1. Let $\Gamma$ satisfy (*). Suppose that $N\left(\Gamma_{x x^{*}}\right)_{x}$ is transitive on the points moved by $\Gamma_{x x^{\prime}}$. Then $\Gamma$ is similar to a 2-transitive collineation group of $\operatorname{PG}(d, 2)$ or $\operatorname{AG}(d, k)$ for some $d$.

Proof. First suppose that $\Pi$ is regular on the points not on $X$. As $\hat{\Gamma}_{x}$ is 2-transitive, Corollary 3.5 and Theorem 4.5 imply the result.

Now assume that $\Pi$ is not regular on the points not on $X$. If $y \notin X$ then $X$ and $y$ determine a unique subdesign (with the same $k$ ) to which our previous results apply, as $\Gamma$ is transitive on the ordered triples $(x, y, X)$ with $x \in X$ and $y \notin X$. If this subdesign consists of the points and lines of PG $(2,2)$ or PG $(3,2)$, the Veblen and Young axioms [16] imply that $\mathscr{D}(\Gamma)$ consists of the points and lines of PG $(d, 2)$ for some $d$. We may thus assume that these subdesigns are affine planes $\operatorname{AG}(2, k)$. It follows that $\Gamma_{x X} / \Gamma(X)$ is cyclic if $x \in X$.

Fix $x$ and $X$ and let $\sigma(X)$ be a generator of $\Gamma_{x X} / \Gamma(X)$, regarded as a permutation of $X$. For each $\gamma \in \Gamma_{x}$ let $\sigma\left(X^{\gamma}\right)=\sigma(X)^{\gamma}$. This is well-defined and independent of the choice of $X$ on $x$. Now set $x^{\sigma}=x$ and, if $y \neq x, y^{\sigma}=y^{\sigma(x y)}$, where $x y$ is the line joining $x$ and $y$. As $\sigma$ fixes each plane containing $x$ and induces a collineation on that plane, $\sigma$ is an automorphism of $\mathscr{D}(\Gamma)$. We may assume that $\sigma \in \Gamma$. As $\Gamma_{x X}$ centralizes $\sigma$ and is maximal in $\Gamma_{x}, \sigma \in Z\left(\Gamma_{x}\right)$. Then a point $y$ is fixed by exactly one conjugate $\sigma_{y}$ of $\sigma=\sigma_{x}$. If $y \neq x$ then $\sigma_{x} \sigma_{y}{ }^{-1}$ fixes each plane $\supset x y$ and has order $p$ on that plane, where $p$ is the prime dividing $k$. Thus, $\sigma_{x} \sigma_{y}{ }^{-1}$ has order $p$. Similarly, there is a unique point $z \in x y$ such that $\sigma_{x} \sigma_{y}{ }^{-1} \sigma_{x} \sigma_{z}{ }^{-1}$ fixes $x y$ pointwise and fixes each plane $\supset x y$. Then $\sigma_{x} \sigma_{y}^{-1} \sigma_{x}=\sigma_{z}$. 
For fixed $x$ let $\Sigma$ be the group generated by the automorphisms $\sigma_{x}^{-1} \sigma_{y}, y \neq x$. We claim that $\Sigma$ is a $p$-group. If $p>2$ this follows from the above remarks and a result of Glauberman [7; Theorem 15]. If $p=2$, then

$$
\sigma_{x}^{-1} \sigma_{y} \cdot \sigma_{x}^{-1} \sigma_{z}=\sigma_{y}^{-1} \sigma_{z}=\sigma_{z}^{-1} \sigma_{y}=\sigma_{x}^{-1} \sigma_{z} \cdot \sigma_{x}^{-1} \sigma_{y}
$$

As $\Sigma$ is transitive on points and is normalized by $\Gamma_{x}$, it is normal in $\Gamma$. Then $\Sigma$ is elementary abelian and regular. As $\sigma_{x}$ has order $k-1$ and is in the centre of $\Gamma_{x}, \Gamma_{x}$ may be regarded as a group of $\mathrm{GF}(k)$-linear mappings. The result now follows.

We note that, once it is assumed that all the subdesigns considered in the above proof are affine planes, the theorem can also be deduced from a result of Bruck [5; p. 100] and a recent result of Buekenhout [3].

CoRollary 5.2. Let $\Gamma$ satisfy $\left({ }^{*}\right)$ with $v$ or $k$ even. If $N\left(\Gamma_{x x^{\prime}}\right)$ is transitive on the points moved by $\Gamma_{x x^{\prime}}$, then $\Gamma$ is similar to a 2-transitive collineation group of $\mathrm{AG}(d, k)$ for some $d$.

Proof. As in the previous proof, if $y \notin X$ then $X$ and $y$ determine a unique subdesign $\mathscr{D}^{*}$. The automorphism group $\Gamma^{*}$ of $\mathscr{D}^{*}$ induced by $\Gamma$ has the property that it is transitive on non-incident point-line pairs.

We first show that $\Gamma^{*}$ is 2-transitive on $\mathscr{D}^{*}$; the proof is essentially that of Ostrom [15]. Let $\Gamma_{x}^{*}$ have $t$ orbits of points $\neq x$ and $t$ orbits of lines on $x$. As each such point-orbit determines such a line-orbit, we have $t^{\prime} \leq t$. However, $\Gamma_{x}^{*}$ has $t+1$ point-orbits and $t^{\prime}+1$ line-orbits, so that $t+1 \leq t^{\prime}+1$ [5; p. 78]. Thus, $\Gamma_{x X}^{*}$ is transitive on $X-\{x\}$. It follows that $\Gamma_{X}^{*}$ is 2-transitive on $X$. Combined with the line-transitivity of $\Gamma^{*}$, this yields the 2-transitivity of $\Gamma^{*}$.

As $(k-1) \mid(v-1)$, by hypothesis $k$ is even. Thus, Theorem 3.4 applies to $\Gamma^{*}$ (acting on $\left.\mathscr{D}^{*}\right)$. By a result of Huppert [11], $\Gamma^{*}$ is non-solvable, so that $\mathscr{D}^{*}$ is $\operatorname{AG}(2, k)$ and $\Gamma^{*}$ satisfies the hypotheses of Theorem 5.1. In view of the transitivity of $\Gamma$ on non-incident point-line pairs, $\Gamma$ also satisfies the hypotheses of Theorem 5.1, and the corollary follows.

Added in proof: While this paper waited almost $2 \frac{1}{2}$ years to be refereed, K. Harada proved and published results which generalize our results of Sections 3 and 4 ("On some doubly transitive groups ", J. Algebra, 17 (1971), 437-450). Harada assumes $(*)$, but replaces $\left(^{* *}\right)$ by the much weaker condition that each involution in $\Gamma_{x x^{\prime}}$ fixes only $k$ points. His methods are group theoretic and not geometric.

Using Harada's results, it is possible to prove the following strengthened version of the results of Section 5. Let $\Gamma$ satisfy $\left(^{*}\right)$, and suppose that $N\left(\Gamma_{x x^{\prime}}\right)$ is transitive on the points moved by $\Gamma_{x x^{\prime}}$. Then either $\mathscr{D}(\Gamma)$ is $\operatorname{PG}(d, 2)$ or $\operatorname{AG}(d, k)$ for some $d$, or $\mathscr{D}(\Gamma)$ is an affine translation plane of odd order (and $\Gamma$ contains the translation group as a regular normal subgroup).

\section{References}

1. H. Bender, "Endliche zweifach transitive Permutationsgruppen, deren Involutionen keine Fixpunkte haben", Math. Z., 104 (1968), 175-204.

2. _- "Transitive Gruppen gerader Ordnung, in denen jede Involution genau einen Punkt festlässt ", J. Algebra, 17 (1971), 527-554.

3. F. Buekenhout, "Une caractérisation des espaces affins basée sur la notion de droite", Math. Z., 111 (1969), 367-371.

4. R. Brauer and Chin-Han Sah, Theory of finite groups (Benjamin, New York, 1969). 
5. P. Dembowski, Finite geometries (Springer, Berlin, 1968).

6. W. Feit and J. G. Thompson, "Solvability of groups of odd order ", Pacific J. Math., 13 (1963), 775-1029.

7. G. Glauberman, "On loops of odd order II ", J. Algebra, 8 (1968), 393-414.

8. D. Gorenstein and J. H. Walter, "The characterization of finite groups with dihedral Sylow 2-subgroups I', J. Algebra, 2 (1965), 85-151.

9. M. Hall, Jr., “Automorphism groups of Steiner triple systems”, Proc. Symp. Pure Math., 6 (1962), 47-66.

10. I. N. Herstein, Topics in algebra (Academic Press, New York, 1964).

11. B. Huppert, "Zweifach transitive auflösbare Permutationsgruppen ", Math. Z., 68 (1957), $126-150$.

12. N. Ito, "On doubly transistive groups of degree $n$ and $\operatorname{order} 2(n-1) n$ ", Nagoya Math. J., 27 (1966), 409-417.

13. —_, "Jordan groups of simplest type" (unpublished manuscript).

14. W. M. Kantor, “Elations of designs ”, Can. J. Math., 22 (1970), 897-904.

15. T. G. Ostrom, " Dual transitivity in finite projective planes ", Proc. Amer. Math. Soc., 9 (1958), $55-56$.

16. Veblen and Young, Projective geometry I (Ginn, Boston, 1911).

\section{University of Illinois}

Chicago, Illinois 